\title{
AVALIAÇÃO DAS PROPRIEDADES FISÍCO-QUÍMICAS DE LINGUIÇA FRESCAL SUÍNA TIPO CAIPIRA COM ADIÇÃO DE HORTELÃ DESIDRATADA
}

\author{
A. C. SOUZA ${ }^{1}$, A. G. S. MOURA ${ }^{1}$ e M. LIMA $^{1}$ \\ ${ }^{1}$ Universidade Federal de Uberlândia, Faculdade de Engenharia Química \\ E-mail para contato: marieli@ufu.br
}

\begin{abstract}
RESUMO - O objetivo do trabalho foi avaliar a influência da hortelã desidratada nas propriedades físico-químicas na linguiça frescal suína tipo caipira armazenada a $4{ }^{\circ} \mathrm{C}$, durante 10 dias. Para isso foram elaboradas três formulações de linguiça suína: (1) controle - alho e sal na formulação, (2) adição de hortelã desidratada e (3) adição de hortelã e sal de cura comercial. Determinaram-se pH, umidade, cinzas, proteína, lipídios e perda de água por cocção. Os dados obtidos através da caracterização físico-química durante o armazenamento foram submetidos à análise de variância e comparação de médias através do Teste T. Os resultados dos parâmetros físico-químicos como lipídios, proteínas e umidade atenderam aos limites previstos pela Legislação Brasileira e não apresentaram diferença significativa entre as formulações testadas $(p>0,05)$. A presença de hortelã e dos sais de cura exerceram alterações significativas no $\mathrm{pH}$ e cinzas das linguiças elaboradas. Durante o armazenamento, observou-se que a formulação (3) demonstrou menos alterações nas suas propriedades físico-químicas durante o período de armazenamento, o que evidencia a importância de se utilizar conservantes para a comercialização deste produto cárneo na forma refrigerada.
\end{abstract}

\section{INTRODUÇÃO}

Segundo Massaguer (2005) a linguiça frescal se sobressai dentre os produtos cárneos embutidos por sua comercialização e aceitação pelos consumidores. $\mathrm{O}$ processo de produção utiliza carnes de animais de açougue adicionadas ou não de tecidos adiposos e a fabricação pode ocorrer em estabelecimentos de micro, pequeno, médio e grande porte. No processamento, agregam-se aditivos e ingredientes utilizados para melhorar as características sensoriais e aumentar o tempo de vida útil do produto.

De acordo com Martins (2007) a linguiça frescal tipo caipira é um produto regional muito apreciado no estado de Minas Gerais, confeccionada de forma artesanal e comercializada para consumo rápido, devido à sua alta perecibilidade. Entretanto, para viabilizar o processamento em escala maior e o armazenamento para comercialização por outros estabelecimentos se faz necessário o uso obrigatório de sais de cura.

Os trabalhos de Pushpangadan e Tewari (2006) demonstraram que a hortelã é um dos condimentos que possui potencial de aplicação na indústria de alimentos, sendo que é uma erva aromática que pode ser usada fresca, seca, como extrato ou como óleo. A secagem de hortelã é um método eficaz que aumenta a vida útil do produto final diminuindo o 
crescimento de microrganismos e protegendo de reações bioquímicas que podem alterar as características sensoriais do produto.

Os trabalhos de Popper et al. (2000) mostraram que de acordo com Legislação Brasileira para embutidos não submetidos ao cozimento, como é o caso da linguiça frescal, é permitida a adição de água ou gelo até o máximo de $3 \%$ na formulação, calculada sobre o total dos componentes e com a finalidade de facilitar a trituração e homogeneização da massa. A linguiça do tipo frescal apresenta como características físico-químicas, umidade máxima de $70 \%$, gordura máxima de $30 \%$, proteína mínima de $12 \%$ e cálcio base seca máximo de $0,1 \%$.

O objetivo deste trabalho é avaliar a influência da hortelã desidratada nas propriedades físico-químicas na linguiça frescal suína tipo caipira.

\section{MATERIAL E MÉTODOS}

$\mathrm{Na}$ elaboração das formulações de linguiça frescal foi utilizado pernil suíno moído refrigerado a $4{ }^{\circ} \mathrm{C}$ e para o embutimento utilizou-se envoltório natural de carneiro. Os ingredientes utilizados na elaboração das linguiças foram alho fresco, cloreto de sódio, açúcar, hortelã desidratada e sais de cura (nitrito, nitrato e cloreto de sódio).

\subsection{Formulação da Linguiça Frescal}

Foram desenvolvidas três formulações diferentes, sendo: controle (Formulação 1); com adição de hortelã desidratada (Formulação 2), e com sal de cura acrescida de hortelã desidratada (Formulação 3).

A matéria-prima e os demais ingredientes utilizados nas três formulações com suas respectivas quantidades estão apresentados na Tabela 1.

Tabela 1 - Descrição das proporções e dos ingredientes utilizados na elaboração da linguiça suína frescal tipo caipira.

\begin{tabular}{|c|c|c|c|c|}
\hline Ingrediente & Quantidade & Controle & Hortelã & Hortelã + sal de cura \\
\hline \hline Carne suína & $1 \mathrm{~kg}$ & $1 \mathrm{~kg}$ & $1 \mathrm{~kg}$ & $1 \mathrm{~kg}$ \\
\hline Sal $(\mathrm{NaCl})$ & $25 \mathrm{~g}$ & $25 \mathrm{~g}$ & $25 \mathrm{~g}$ & $25 \mathrm{~g}$ \\
\hline Alho fresco & $3 \mathrm{~g}$ & $3 \mathrm{~g}$ & $3 \mathrm{~g}$ & $3 \mathrm{~g}$ \\
\hline Água gelada & $200 \mathrm{~mL}$ & $200 \mathrm{~mL}$ & $200 \mathrm{~mL}$ & $200 \mathrm{~mL}$ \\
\hline $\begin{array}{c}\text { Hortelã } \\
\text { desidratada }\end{array}$ & $5 \mathrm{~g}$ & --- & $5 \mathrm{~g}$ & $5 \mathrm{~g}$ \\
\hline Açúcar & $1 \mathrm{~g}$ & --- & $1 \mathrm{~g}$ & $1 \mathrm{~g}$ \\
\hline Sal de cura & $5 \mathrm{~g} *$ & --- & -- & $5 \mathrm{~g}$ \\
\hline
\end{tabular}

*Recomendação do fabricante.

\subsection{Análises Físico-Químicas}

As amostras foram avaliadas ao longo dos 10 dias de armazenamento a $4{ }^{\circ} \mathrm{C}$, nos tempos: 0, 5 e 10 dias. Foram determinados $\mathrm{pH}$, umidade, cinzas, proteínas e lipídios através 
das normas do Instituto Adolfo Lutz (2009). A perda de água por cocção foi determinada segundo a metodologia adaptada de AMSA (1995).

\subsection{Análise Estatística}

Os dados de caracterização físico-química da linguiça frescal foram submetidos à análise de variância (ANOVA) e comparações de médias (teste de T), para verificar se havia diferença significativa entre as formulações utilizadas e se houve mudanças nos parâmetros físico-químicos com tempo de armazenamento. Para a realização da análise de variância e teste T foi utilizado o software Assistat 7.7 beta (versão gratuita).

\section{RESULTADOS E DISCUSSÃO}

Os valores encontrados para o $\mathrm{pH}$ (Tabela 2), as linguiças diferiram estatisticamente quanto ao tipo de formulação e quanto ao tempo de armazenamento. De acordo com a Legislação Brasileira não há limites de pH pré-estabelecidos para a linguiça frescal, apenas menciona que o pH deverá ser levemente ácido.

Segundo Mantovani et al. (2011) os valores considerados como normais de pH para produtos cárneos oscilam entre 5,4 e 6,2. Desta maneira, os valores de $\mathrm{pH}$ das linguiças neste estudo encontram-se dentro da normalidade pois variaram de 5,50 a 5,83. De acordo com Milani et al. (2003), quanto mais elevado o pH, maior é a probabilidade de desenvolvimento microbiano. No presente estudo, em 10 dias de armazenamento houve uma redução no valor do $\mathrm{pH}$ de 5,75 para 5,50 na linguiça acrescida de hortelã e sal de cura, fazendo com que o produto ficasse com menor probabilidade de desenvolvimento de microrganismos e consequentemente maior estado de conservação.

Tabela 2 - Média e desvio padrão do pH das três formulações de linguiça frescal suína tipo caipira durante os dez dias de armazenamento a $4^{\circ} \mathrm{C}$.

\begin{tabular}{|c|c|c|c|}
\hline \multirow{2}{*}{$\begin{array}{c}\text { Tempo } \\
\text { (dias) }\end{array}$} & \multicolumn{3}{|c|}{$\mathrm{pH}$} \\
\cline { 2 - 4 } & Controle & Hortelã & Hortelã + Sal de cura \\
\hline 00 & $5,75 \pm 0,08^{\mathrm{aA}}$ & $5,75 \pm 0,04^{\mathrm{aA}}$ & $5,75 \pm 0,05^{\mathrm{aA}}$ \\
\hline 05 & $5,65 \pm 0,04^{\mathrm{abB}}$ & $5,61 \pm 0,09^{\mathrm{aB}}$ & $5.79 \pm 0,20^{\mathrm{bA}}$ \\
\hline 10 & $5,83 \pm 0,04^{\mathrm{aC}}$ & $5,71 \pm 0,04^{\mathrm{bA}}$ & $5,50 \pm 0,08^{\mathrm{aB}}$ \\
\hline
\end{tabular}

A Tabela 3 apresenta as médias dos valores de umidade, visto que não diferiram estatisticamente entre os processos. Porém, em relação ao tempo de armazenamento houve diferença significativa para as três formulações. As médias de umidade das linguiças obtidas neste trabalho encontram-se na faixa de 66,41 a $69,73 \%$, cujos resultados estão dentro dos padrões físico-químicos estabelecidos pelo Regulamento Técnico de Identidade e Qualidade de Linguiças, que de acordo com Brasil (2000), é estabelecido um máximo de $70 \%$ de umidade para linguiças frescais.

O aumento no conteúdo de umidade em relação ao tempo de armazenamento nos diferentes processos pode ser proveniente de alterações no produto promovidas por atividade enzimática, como proteólise e ação microbiana. É possível que no decorrer do período o teor de água livre decorrente das alterações físico-químicas e microbiológicas do produto tenha 
aumentado, por este motivo no quinto dia de armazenamento era possível observar visualmente uma exsudação aparente nas linguiças.

Tabela 3 - Média da umidade no processamento de linguiça frescal suína tipo caipira nos três tratamentos durante os dez dias de armazenamento a $4^{\circ} \mathrm{C}$.

\begin{tabular}{|c|c|c|c|}
\hline \multirow{2}{*}{$\begin{array}{c}\text { Tempo } \\
(\text { dias })\end{array}$} & \multicolumn{3}{|c|}{ Umidade $(\%)$} \\
\cline { 2 - 4 } & Controle & Hortelã & Hortelã + Sal de cura \\
\hline 00 & $66,41 \pm 2,08^{\mathrm{aA}}$ & $67,97 \pm 1,86^{\mathrm{aA}}$ & $65,19 \pm 3,21^{\mathrm{aA}}$ \\
\hline 05 & $68,54 \pm 3,58^{\mathrm{abA}}$ & $68,50 \pm 1,36^{\mathrm{abA}}$ & $69,33 \pm 0,29^{\mathrm{bA}}$ \\
\hline 10 & $69,66 \pm 0,21^{\mathrm{bA}}$ & $69,57 \pm 0,23^{\mathrm{bA}}$ & $69,71 \pm 0,27^{\mathrm{bA}}$ \\
\hline
\end{tabular}

A Tabela 4 apresenta as médias das triplicatas da determinação de cinzas, proteínas e lipídeos. O percentual de cinzas encontrado para as linguiças diferiu estatisticamente entre si ( $\mathrm{p}<0,05)$, visto que as médias de cinzas obtidas neste trabalho encontram-se na faixa de 2,83 a $3,05 \%$. O conteúdo de cinzas aumenta com a adição de alguns condimentos utilizados no preparo de linguiça. Os maiores valores de resíduo mineral encontrado foram nas linguiças com adição de hortelã e acrescida de hortelã mais sal de cura, o que influenciou diretamente no aumento do teor de cinzas.

Os resultados do teor de proteína das linguiças frescais nos diferentes tratamentos não diferiram significativamente entre si ( $>00,05)$. De acordo com Brasil (2000) o valor de proteínas estabelecido pelo Regulamento Técnico de Identidade e Qualidade para linguiças frescais é o mínimo de $12 \%$. As médias do teor de proteínas das linguiças obtidas neste trabalho encontraram-se na faixa de 19,09 a $19,18 \%$, superiores ao padrão mínimo permitido pela Legislação Brasileira. Os trabalhos de Silva et al. (2012) apresentaram 18\% de proteína para a linguiça frescal suína, compatível com o conteúdo de proteína obtida neste trabalho.

Os valores de lipídeos obtidos neste estudo variaram de 0,44 a $0,54 \%$, visto que não houve diferença significativa entre os tratamentos ( $>0,05$ ). Os resultados estão de acordo com a Legislação Brasileira (Brasil, 2000) onde é estabelecido um máximo de $30 \%$ de lipídeos para linguiças frescais. Todos os tratamentos apresentaram conteúdo lipídico dentro do limite máximo permitido pela Legislação Brasileira. Isso pode ser explicado pelo fato de que à matéria-prima utilizada nas três formulações não foram acrescidas de toucinho, que promove um acréscimo no conteúdo lipídico da linguiça, conforme obtido por Silva et al. (2012), que utilizaram toucinho na elaboração de linguiça frescal e obtiveram teor de lipídeos em torno de 10,98\%. Desta maneira, pode considerar que as linguiças controle, hortelã e hortelã acrescida com sal de cura possuem baixo conteúdo de gordura.

A Tabela 5 apresenta os valores de perda de água por cocção. As médias obtidas neste trabalho encontram-se na faixa de 45,84 a 56,86\%, sendo que não houve diferença significativa entre os processos no período inicial e aos dez dias de armazenamento. Por outro lado, no quinto dia houve diferença significativa entre os processos.

No período inicial, a perda de água por cocção foi semelhante nos diferentes processos. Contudo, a perda de peso durante o cozimento indicou percentuais acima das demais para a linguiça acrescida de sal de cura. Tal situação pode ser entendida a partir da compreensão do papel do sal na retenção de água da carne. 
Tabela 5 - Média da perda de água por cocção no processamento de linguiça frescal suína tipo caipira nos três tratamentos durante os dez dias de armazenamento a $4^{\circ} \mathrm{C}$.

\begin{tabular}{|c|c|c|c|}
\hline \multirow{2}{*}{$\begin{array}{c}\text { Tempo } \\
\text { (dias) }\end{array}$} & \multicolumn{3}{|c|}{ Perda de água por cocção $(\%)$} \\
\cline { 2 - 4 } & Controle & Hortelã & Hortelã + Sal de cura \\
\hline 00 & $47,81 \pm 1,97^{\mathrm{aA}}$ & $48,13 \pm 5,56^{\mathrm{aA}}$ & $48,76 \pm 3,06^{\mathrm{aA}}$ \\
\hline 05 & $45,84 \pm 1,64^{\mathrm{aA}}$ & $46,09 \pm 3,35^{\mathrm{aA}}$ & $56,86 \pm 3,91^{\mathrm{bB}}$ \\
\hline 10 & $50,22 \pm 7,01^{\mathrm{aA}}$ & $47,79 \pm 4,53^{\mathrm{aA}}$ & $53,37 \pm 8,26^{\mathrm{aAB}}$ \\
\hline
\end{tabular}

Em relação ao tempo de armazenamento as médias de perda de água por cocção não diferiram estatisticamente ao longo do tempo para as formulações controle e com adição de hortelã. Porém para a linguiça com hortelã acrescida de sal de cura, houve diferença significativa. Desta maneira, foi possível observar que as linguiças com adição de hortelã e sal de cura apresentaram maiores perdas de água por cocção. No entanto, para as linguiças onde houve a adição exclusiva de hortelã houve menores perdas de água após dez dias de armazenamento. Acredita-se que a hortelã contribuiu como coadjuvante na capacidade de retenção de água das linguiças.

\section{CONCLUSÕES}

A caracterização físico-química das três formulações testadas demonstrou que o produto foi desenvolvido dentro dos critérios previstos pelo Regulamento Técnico de Identidade e Qualidade de Linguiça, do MAPA. A presença de hortelã e dos sais de cura influenciou no pH e no teor de cinzas, que apresentaram diferenças significativas entre as formulações. O teor de proteínas, lipídeos e umidade não foram afetados pela presença de hortelã e pelos sais de cura, preservando os parâmetros de identidade da linguiça frescal.

O armazenamento da linguiça frescal suína tipo caipira sob refrigeração evidenciou exsudação visível nas embalagens estocadas após alguns dias de armazenamento. Conforme era esperado, a Formulação (3) demonstrou menos alterações nas suas propriedades físicoquímicas durante o período de armazenamento, o que evidencia que a presença de condimentos e dos sais de cura contribuíram para a estabilidade do produto e podem contribuir para a comercialização deste produto cárneo na forma refrigerada.

Para a determinação da vida útil e da viabilidade deste produto para comercialização, é necessário um estudo complementar sobre avaliação microbiológica, bem como uma avaliação sensorial a fim de se determinar a aceitabilidade dos consumidores.

\section{REFERÊNCIAS}

AMSA (1995). Research guideliner for cookery, sensory evaluation and instrumental tenderness measurements of fresh meat. National Live-stock and Meat Bard, Chicago. 
BRASIL. Ministério da Agricultura, Pecuária e do Abastecimento. Secretaria de Defesa Agropecuária. Departamento de Inspeção de Produtos de Origem Animal. Regulamento da Inspeção. Instrução Normativa ${ }^{\circ} 4$, de 31 de março de 2000. Regulamento Técnico de Identidade e Qualidade de lingüiça. Anexo III - Regulamento Técnico de Identidade e Qualidade de Linguiça. Diário Oficial da União, Brasília, DF, 05 abr. 2000.

INSTITUTO ADOLFO LUTZ. Normas Analíticas do Instituto Adolfo Lutz. v. 1: Métodos químicos e físicos para análise de alimentos. 4. ed. São Paulo, 2009.

MANTOVANI, D.; CORAZZA, M. L.; CARDOZO FILHO, L.; COSTA, S. C. Avaliação higiênico-sanitária de linguiças tipo frescal após inspeção sanitária realizada por órgãos federal, estadual e municipal na região noroeste do Paraná. Revista Saúde e Pesquisa, v.4, n.3, p.357-362, 2011.

MARTINS, R. Dossiê Técnico. Produção de linguiça frescal. Rio de Janeiro, 2007. Disponível em: http://www.sbrt.ibict.br/dossie-tecnico/downloadsDT/MTQy Acesso em: 27 de mar. 2017.

MASSAGUER, P. Microbiologia dos processos alimentares. São Paulo: Varela, 2005.

MILANI, L. I. G.; FRIES, L. L. M; PAZ, P. B.; BELLÉ, M.; TERRA, N. N. Bioproteção de linguiça de frango. Ciência e Tecnologia de Alimentos, v.23, n.2, p.161-166, 2003.

POPPER, I.; CARLOS, M.A.; FIGUEIREDO, B.; GARCIA, S.; PINTO, M.P.; SILVA, A.C.; SOUSA, I.F.; PRIMO, C.B.B.; BORGES, R. Avaliação da formulação de linguiças tipo frescal coletadas no município de Londrina, quanto aos teores de gordura, proteína e água. Congresso Brasileiro de Ciência e Tecnologia de Alimentos, 2000, Fortaleza. Anais: Ceará, 2000.

PUSHPANGADAN, P.; TEWARI, S. K.. Peppermint. India. In: PETER, K. V. (Comp.). Handbook of herbs and spices. Boca Raton, Fla.; Cambridge, England: CRC: Woodhead Pub. nv. (Woodhead Publishing in food science and technology). Cap. 28. vol. 3.p. 460-481, 2006.

SILVA, F. G. S.; LEÃO, K. M.; BARROS, J. C.; SILVA, B; GIOVANNINI, C. I.; SILVA, M. A. P. Composição centesimal e aceitabilidade de linguiça frescal de carne caprina. In: Congresso de Pesquisa e Pós-Graduação do Campus Rio Verde do IF Goiano. 06 e 07 de novembro de 2012. 